\title{
Some Traditional Medicinal Plants Useful for Boil, Burn and for Wounds Healing
}

\section{Patel DK*}

Department of Rural Technology, Guru Ghasidas Vishwavidyalaya, (A Central University), Bilaspur, 495009, Chhattisgarh, India

\begin{abstract}
Traditional Medicinal plants are remarkable for their significant potential to treat a specific disorder. Over the world around $80 \%$ populations dependent on traditional medicines according to WHO. Plant diversity depends on local climatic condition and also on their adaptation capacity in changeable environmental condition. Plants are great source of primary health care due to presence of certain chemical compounds. Cut, Boil, Burn, Wounds are mainly affecting skin. Prevention of pathogenic attack in body plants can perform certain role known as herbal medicine. A total of 55 species of the plants of 35 different families were recorded for above purpose and the findings are listed and discussed.
\end{abstract}

Keywords: Boil burn; Herbal plants; Plant diversity; Wound

\section{Introduction}

Plants are used for treatment of certain disorders from a long ago [1]. Plants registered as a major source of medicinal preparations and also many drugs derived from herbal plants [2]. Around 25\% drugs are derived by using plants [3]. Due to less hygienic situation mostly in rural areas wounds is common disorder for skin problem [4].

Boil, Burn, wounds are accidental physical damage of body by loss of skin. Use of firewood for cooking and other household activities sometimes being reason for burning of skin [5]. Uses of the plants as a source of medicine for boil, burn wounds are important for healthcare in rural areas.

Wound healing process start from damage of skin. A wound completely healing depends on degree of injury, human resistance capacity, infection potential of pathogens and early effective treatment procedure. Above process completing following several steps events. During cutting of skin it is prime need to stop bleeding from body. Many plants are showing better performance for this purpose like Aloe vera, Tridex procumbens etc. Use of Carica papaya latex for wound healing was noticed by Gurang and Basent, [6]. Wound healing ethno- pharmacological potentials of selected medicinal plants used by malayali tribals was noticed by Subramaninan et al. [7].

A large group of the plants are marked as efficient for boil, burn wounds and for skin related problems [8-10]. Wound healing capacity of papaya (Carica papaya) latex noticed by Basert, [6]. Its fruits are also used for this purpose. Wounds may be chronic or by accidental. It is a physical injury of skin responsible for loss of colour of skin and loss of structure. Applying plants for control of boil, burn wounds are beneficial process for repairing of body. Due to presence of antibiotic or antiseptic nature chemicals plants are remarkable for many disorders [11].

Chemicals derived from plants need for proper identification, formulation, use and also for protection. Traditional knowledge of plants for medicinal uses in rural areas is of significant potential connections between plants and local peoples. Plants providing low cast, efficient, less side effect treatment for certain problems. As an estimation of WHO $80 \%$ peoples of the world depends on herbal medicine. A review on medicinal plants with potential wound healing activity recorded by Kumrasamyraja et al. [12].

Around $70 \%$ of the pharmaceutical products for wound control are made by using plant resources. Wounds can be referred as physical disabilities [13]. Wounds are marked as injury in normal skin structural, anatomical physiological and functional variation [14]. Many plants supporting natural repairing process of skin [15]. Plants are also variable for use in blood coagulation and cleaning of skin/ wounds etc $[16,17]$. Wound healing of some medicinal plants was reviewed by Gulzar et al. [18].

India is rich center of floral diversity which is distributed over the country. Around $25 \%$ of drugs are derived from varied plant species and used as medicinal purpose in rural areas [19]. Healing of wound is essential for filling/ restoring of the cells in affecting area of body [20]. Use of the plants in indigenous traditional medicine system is very old in India [21]. Rural peoples mostly used the plants for treatment of various ailments. Ethno botany is old in India [22-24]. A review on the use of Aloe vera for wound healing was made by Ratree et al. [24]. Ethno botanical study on herbal medicines for wound healing among tribal peoples in southern part of India was made by Ayyar et al. [21]. Medicinal plants used by traditional healers in Kancheepuram district of Tamil nadu India was recorded by Muthu et al. [22].

In India there are 2500 plants having medicinal values and near 6000 plants are used in traditional medicinal system. Rural Indians using traditional medicinal plants for various purpose. The objectives of the present study was to explore the utility of plants among rural peoples for various use especially for Boil, Burn, and for Wounds in nearby the areas of Bilaspur (C. G.) in Central part of Chhattisgarh.

\section{Materials and Methods}

Information related to the use of the plant species were collected in 2011-2012 near the villages of Bilaspur (C. G.). The information was collected by general conversation with knowledgeable persons of the area and plants local name, used parts, propagation etc was known from him. Other scientific plant related information was gained by using literatures.

*Corresponding author: Patel DK, Department of Rural Technology, Guru Ghasidas Vishwavidyalaya, Bilaspur, 495009, Chhattisgarh, India, Tel: 09993660173; E-mail: dplantscience@yahoo.co.in

Received June 04, 2014; Accepted July 17, 2014; Published July 22, 2014

Citation: Patel DK (2014) Some Traditional Medicinal Plants Useful For Boil, Burn and For Wounds Healing. J Biodivers Endanger Species 2: 133. doi:10.4172/2332-2543.1000133

Copyright: @ 2014 Patel DK. This is an open-access article distributed under the terms of the Creative Commons Attribution License, which permits unrestricted use, distribution, and reproduction in any medium, provided the original author and source are credited. 
Citation: Patel DK (2014) Some Traditional Medicinal Plants Useful for Boil, Burn and for Wounds Healing. J Biodivers Endanger Species 2: 133. doi:10.4172/2332-2543.1000133

Page 2 of 4

\section{Results and Discussion}

A variety of plants species are used for different disorders in rural areas. Particularly the plants which are used for boil, burn and for wound are documented/ listed in (Table 1). Table 2 showing family wise number of the plants. Table 3 and Figure 1 is for Habit variation.

\begin{tabular}{|c|c|c|c|c|c|c|}
\hline S.No. & Botanical Name & Common/Name & Family & Habit & Parts Used & Propagation \\
\hline 1. & Adhatoda vasica & Vasaka & Acanthaceae & Shrub & Leaf, soft stem & Stem cutting \\
\hline 2. & Aegle marmelos & Bael & Rutaceae & Tree & Leaf, bark, Fruit & Seed \\
\hline 3. & Ageratum conyzoids & White weed & Asteraceae & Herb & Leaf & Seed \\
\hline 4. & Allium cepa & Onion & Liliaceae & Herb & Bulb & Bulb \\
\hline 5. & Aloe vera & Gwarpatha & Liliaceae & Herb & Leaf & Bud \\
\hline 6. & Annona squmosa & Sitaphal & Annonaceae & Shrub & Leaf, Fruit & Seed \\
\hline 7. & Anthocephalus cadamba & Kadamb & Rubiaceae & Tree & Bark & Seed \\
\hline 8. & Argemone maxicana & Pili kateri & Papavaraceae & Herb (Spiny) & Leaf & Seed \\
\hline 9. & Azadiracta indica & Neem & Meliaceae & Tree & Leaf, Oil & Seed \\
\hline 10. & Blumea lacera & Hul hul & Asteraceae & Herb & Leaf & Seed \\
\hline 11. & Boerhavia diffusa & punarnava & Nyctaginaceae & Herb & Leaf & Seed \\
\hline 12. & Butea monosperma & Palas & Fabaceae & Tree & Bark & Seed \\
\hline 13. & Calotropis gigantia & AK & Asclepiodaceae & Shrub & Leaf, Stem & Seed \\
\hline 14. & Canna indica & Baijanti & Cannaceae & Herb & Stem & Rhizome \\
\hline 15. & Carica papaya & Papita & Caricaceae & Herb & Leaf, Fruit & Seed \\
\hline 16. & Cassia alata & & Fabaceae & Shrub & Leaf & Seed \\
\hline 17. & Cassia tora & Charota & fabaceae & Herb & Leaf & Seed \\
\hline 18. & Centella asiatica & Madukparni & apiaceae & Herb & Leaf & Stem cutting \\
\hline 19. & Chenopodium album & Bathua & Chenopodiaceae & Herb & Leaf & Seed \\
\hline 20. & Cleome viscose & & Cleomaceae & Herb & Leaf & Seed \\
\hline 21. & Clitoria ternatea & Butterfly flower & Fabaceae & Herb & Leaf & Seed \\
\hline 22. & Costus speciosus & Keu kand & Zinziberaceae & Herb & Leaf, Rhizome & Rhizome \\
\hline 23. & Curcuma longa & Haldi & Zinziberaceae & Herb & Rhizome & Rhizome \\
\hline 24. & Daucas carota & Gajar & Apiaceae & Herb & Root & Seed \\
\hline 25. & Diospyrus melanoxylon & Tendu & Ebenaceae & Tree & Fruit & Seed \\
\hline 26. & Eclipta alba & Bhringraj & Asteraceae & Herb & Leaf & Seed \\
\hline 27. & Euphorbia hirta & Dudhi & Euphorbiaceae & Herb & Leaf, Stem & Seed \\
\hline 28. & Ficus bengalensis & bargad & ficaceae & tree & Leaf, Bark & Seed \\
\hline 29. & Gloriosa superva & Kalihari & Liliaceae & Herb & Tuber & Tuber \\
\hline 30. & Helianthus annus & Sun flower & asteraceae & Herb & oil & Seed \\
\hline 31. & Heliotropium indicum & Indialn Turnsole & Boraginaceae & Herb & Leaf & Seed \\
\hline 32. & Hyptis suaveolens & Van Tulsi & Lamiaceae & Herb & Leaf & Seed \\
\hline 33. & Ixora coccnia & Ixora & Rubiaceae & Shrub & Flower & Stem cutting \\
\hline 34. & Lawsonia inermis & Mehandi & Lythraceae & Shrub & Leaf & Seed \\
\hline 35. & Mentha viridis & Podina & Lamiaceae & Herb & Leaf & Stem cutting \\
\hline 36. & Mimosa pudica & Chui-mui & fabaceae & Herb & Leaf & Seed \\
\hline 37. & Moringa oleifera & Munga & Moringaceae & Tree & Leaf, Bark & Seed \\
\hline 38. & Murraya paniculata & Meetha neem & Rutaceae & Shrub & Leaf & Seed \\
\hline 39. & Musa paradisica & Kela & Musaceae & Herb & Stem, Fruit & Rhizome \\
\hline 40. & Nerium indicum & Kaner & apocynaceae & Shrub & Leaf & Seed/Stem cutting \\
\hline 41. & Pongamoea pinnata & Karanj & Fabaceae & Tree & Bark & Seed \\
\hline 42. & Pothos scandens & Money plant & Araceae & Herb & Leaf & Stem cutting \\
\hline 43. & Psidium guava & Amrud & Myrtaceae & Tree & Leaf, Fruit & Seed \\
\hline 44. & Ptephrosia perpuria & Wild indigo & Fabaceae & Herb & Leaf & Seed \\
\hline 45. & Punica granatum & Anar & Punicaceae & Shrub & Bark & Seed \\
\hline 46. & Rosa indica & Rose & Rosaceae & Shrub & Leaf & Stem cutting \\
\hline 47. & Sesamum indicum & Tilli & Pedaliaceae & Herb & oil & Seed \\
\hline 48. & Sida rhombifolia & Arrow leaf sida & Malvaceae & Herb & Leaf & Seed \\
\hline 49. & Solanum indicum & Potato & Solanaceae & Herb & Tuber & Seed \\
\hline 50. & Solanum xanthocarpum & Bhatkatia & Solanaceae & Herb (Spiny) & fruit & Seed \\
\hline 51. & Syzygium cumini & Jamun & Myrtaceae & Tree & Leaf, Bark & Seed \\
\hline 52. & Tagetes patula & Chandani genda & Asteraceae & Herb & Leaf & Seed \\
\hline 53. & Terminalia chebula & Harra & Combrataceae & Tree & Fruit & Seed \\
\hline 54. & Tridex procumbens & Coat button & Asteraceae & Herb & Leaf & Seed \\
\hline 55. & Vitex negundo & Negur & Verbanaceae & Shrub & Leaf & Stem cutting \\
\hline
\end{tabular}

Table 1: Plant species useful for boil, burn and for wounds. 
Citation: Patel DK (2014) Some Traditional Medicinal Plants Useful for Boil, Burn and for Wounds Healing. J Biodivers Endanger Species 2: 133. doi:10.4172/2332-2543.1000133

Page 3 of 4

\begin{tabular}{|c|c|c|c|}
\hline S. No. & Family & No. of plant species & Percentage \\
\hline 1. & Acanthaceae & 1 & 1.82 \\
\hline 2. & Annonaceae & 1 & 1.82 \\
\hline 3. & Apiaceae & 2 & 3.64 \\
\hline 4. & Apocynaceae & 1 & 1.82 \\
\hline 5. & Araceae & 1 & 1.82 \\
\hline 6. & Asclepiodaceae & 1 & 1.82 \\
\hline 7. & Asteraceae & 6 & 10.90 \\
\hline 8. & Boraginaceae & 1 & 1.82 \\
\hline 9. & Cannaceae & 1 & 1.82 \\
\hline 10. & Caricaceae & 1 & 1.82 \\
\hline 11. & Chenopodiaceae & 1 & 1.82 \\
\hline 12. & Cleomaceae & 1 & 1.82 \\
\hline 13. & Combrataceae & 1 & 1.82 \\
\hline 14. & Ebenaceae & 1 & 1.82 \\
\hline 15. & Euphorbiaceae & 1 & 1.82 \\
\hline 16. & Fabaceae & 7 & 1.82 \\
\hline 17. & Ficaceae & 1 & 1.82 \\
\hline 18. & Lamiaceae & 2 & 3.64 \\
\hline 19. & Liliaceae & 3 & 5.45 \\
\hline 20. & Lythraceae & 1 & 1.82 \\
\hline 21. & Malvaceae & 1 & 1.82 \\
\hline 22. & Meliaceae & 1 & 1.82 \\
\hline 23. & Moringaceae & 1 & 1.82 \\
\hline 24. & Musaceae & 1 & 1.82 \\
\hline 25. & Myrtaceae & 2 & 3.64 \\
\hline 26. & Nyctaginaceae & 1 & 1.82 \\
\hline 27. & Papavaraceae & 1 & 1.82 \\
\hline 28. & Pedaliaceae & 1 & 1.82 \\
\hline 29. & Punicaceae & 1 & 1.82 \\
\hline 30. & Rosaceae & 1 & 1.82 \\
\hline 31. & Rubiaceae & 2 & 3.64 \\
\hline 32. & Rutaceae & 2 & 3.64 \\
\hline 33. & Solanaceae & 2 & 3.64 \\
\hline 34. & Verbanaceae & 1 & 1.82 \\
\hline 35. & Zinziberaceae & 2 & 3.64 \\
\hline \multicolumn{2}{|l|}{ TOTAL } & 55 & \\
\hline
\end{tabular}

Table 2: Plant species useful for boil, burn and for wounds.

\begin{tabular}{|c|l|l|l|}
\hline S. No. & Habit & Number of the Plants & Percentage \\
\hline 1. & Herb & 31 & 56.36 \\
\hline 2. & Herb (Spiny) & 02 & 3.64 \\
\hline 3. & Shrub & 11 & 20.00 \\
\hline 4. & Tree & 11 & 20.00 \\
\hline TOTAL & & $\mathbf{5 5}$ & \\
\hline
\end{tabular}

Table 3: Habit of the Plant species.

Table 4 and Figure 2 plant parts used and Table 5 and Figure 3 is for propagation mode of the plants. A total of 55 species of plants belonging 35 families were recorded.

On the basis of habit 32 herbs 11 shrubs and 12 trees were found to be useful for this purpose. As used plant parts maximum leaf part is recorded for the above purpose. Propagation method maximum $72.72 \%$ recorded for Seeds. Plants were recorded as their external use in treatment. Recorded plant species were of varied families like one members from each families like Acanthaceae, Annonaceae , Apocynaceae, Araceae, Asclepiodaceae, Boraginaceae, Cannaceae, Caricaceae, Chenopodiaceae, Cleomaceae, Combrataceae, Ebenaceae, Euphorbiaceae, Ficaceae, Lythraceae, Malvaceae, Meliaceae, Moringaceae, Musaceae, Nyctaginaceae, Papavaraceae, Pedaliaceae,
Punicaceae, Rosaceae, Verbanaceae. Two members from Apiaceae, Lamiaceae, Myrtaceae, Rubiaceae, Rutaceae, Solanaceae, Zinziberaceae. Three members from Liliaceae were recorded and six members from family Asteraceae were recorded.

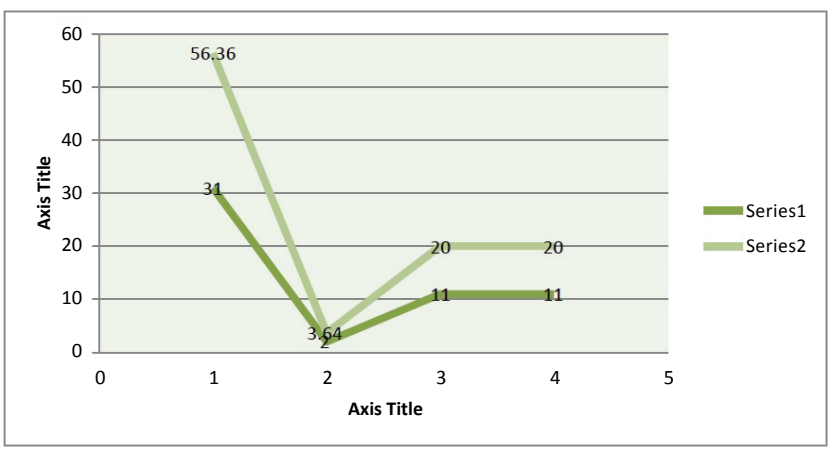

Figure 1: Graphical representation of Habit Plant species.

\begin{tabular}{|c|l|l|l|}
\hline S. No. & Parts Used & Number of the Plants & Percentage \\
\hline 1. & Bark & 4 & 7.27 \\
\hline 2. & Bulb & 1 & 1.82 \\
\hline 3. & Flower & 1 & 1.82 \\
\hline 4. & Fruit & 3 & 5.45 \\
\hline 5. & Leaf & 26 & 47.27 \\
\hline 6. & Leaf, Bark & 3 & 5.45 \\
\hline 7. & Leaf, bark, Fruit & 1 & 1.82 \\
\hline 8. & Leaf, Fruit & 3 & 5.45 \\
\hline 9. & Leaf, Rhizome & 1 & 1.82 \\
\hline 10. & Leaf, Stem & 3 & 5.45 \\
\hline 11. & Leaf. Oil & 1 & 1.82 \\
\hline 12. & oil & 2 & 3.64 \\
\hline 13. & Rhizome & 1 & 1.82 \\
\hline 14. & Root & 1 & 1.82 \\
\hline 15. & Stem & 1 & 1.82 \\
\hline 16. & Stem, Fruit & 1 & 1.82 \\
\hline 17. & Tuber & 2 & 3.64 \\
\hline TOTAL & & 55 & \\
\hline & & & \\
\hline
\end{tabular}

Table 4: Plant Parts used.

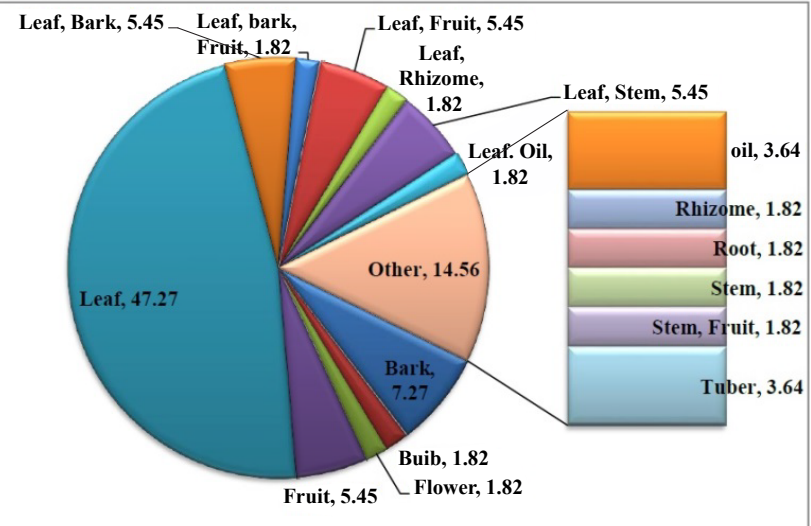

Figure 2: Graphical representation of Plant Parts used. 
Citation: Patel DK (2014) Some Traditional Medicinal Plants Useful for Boil, Burn and for Wounds Healing. J Biodivers Endanger Species 2: 133. doi:10.4172/2332-2543.1000133

\begin{tabular}{|c|l|l|l|}
\hline S. No. & Propagation & No. of plants & Percentage \\
\hline 1. & Bud & 1 & 1.82 \\
\hline 2. & Bulb & 1 & 1.82 \\
\hline 3. & Rhizome & 4 & 7.27 \\
\hline 4. & Seed & 40 & 72.72 \\
\hline 5. & Seed/Stem cutting & 1 & 1.82 \\
\hline 6. & Stem cutting & 7 & 12.72 \\
\hline 7. & Tuber & 1 & 1.82 \\
\hline TOTAL & & $\mathbf{5 5}$ & \\
\hline
\end{tabular}

Table 5: Propagation mode of the Plants.

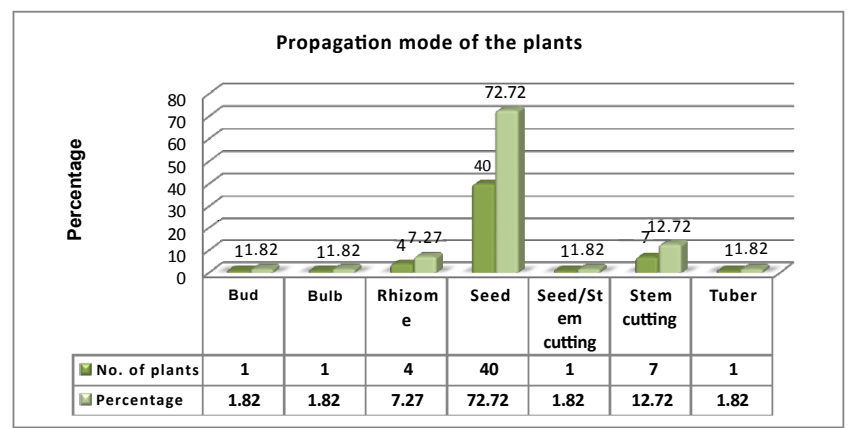

Figure 3: Graphical representation of Propagation mode of the Plants.

\section{References}

1. Chah KF, Eze CA, Emuelosi CE, Esimone CO (2006) Antibacterial and wound healing properties of methanolic extracts of some Nigerian medicinal plants. $\mathrm{J}$ Ethnopharmacol 104: 164 -167.

2. Fabricant DS, Farnsworth NR (2001) The value of plants used in traditional medicine for drug discovery Environ Health Pers 109: 69-75.

3. Principe $P$ (2005) Monetising the pharmacological benefits of plants. US Environmental protection Agency, Washington DC, 1991.

4. Senthil KM, Sripriya R, Vijaya RH, Sehgal P (2006) Wound Healing Potential of Cassia fistula on Infected Albino Rat Model. J Surg Res 131: 283-289.

5. Anonymous, HMG, (2003) The Tenth Plan (Poverty Reduction Strategy Paper) 2002-2007, National Planning Commission, Kathmandu.

6. Gurung S, Basent NS (2009) Wound healing properities of Carica papaya latex : In vivo evaluation in mice burn model. Journal of Ethnopharmacology 121: 338- 41.

7. Subramanian R, Krishnasamy G, Devraj A, Sethuraman $P$, Jayakumararaj $R$ (2011) Wound healing ethnopharmacological potential of selected Medicinal Plants used by malayali tribes, International Research Journal of Pharmacy 2: $132-137$
8. Muthu C, Ayyanar M, Raja N, Ignacimuthu S (2006) Medicinal plants used by traditional healers in Kancheepuram District of Tamil nadu India, 2: 1-10.

9. Singh M, Govindarajan R, Nath V, Rawat AKS, Mehrotra S (2006) Antimicrobial wound healing and antioxidant activity of Plagiochasma appendiculatum Lehm. et Lind. Journal of Ethnopharmacology 107: 67-72.

10. Savanth SS, Shah RA (1998) In Savant SS, Shah RA, Gore D. Text book and atlas of Dermatology and Cosmetology, Mumbai: ASCAD; 12-17

11. Chulani HL (1996) In The law of medical negligence 1st edn. Radhakrishan Medical and Educational Trust: Mumbai 51-83.

12. Kumarasamyraja D, Jeganathan NS, Manavalan R (2012) A review on Medicinal plants with potential wound healing activity, International Journal of Pharma Sciences 4:105 - 111.

13. F Strodtbeck (2001) Physiology of wound healing, new born infant nurse, 43-45

14. Chitra S, Patil MB, Ravikumar (2009) Preliminary phytochemical investigation and wound healing activity of Allium.Cepalin (Liliaceae). International journal of pharmacy and pharmaceutical sciences.

15. Kumar BM, Govindarajan R, Pusphagandan (2007) Ethanopharmacological approaches to wound healing- Exploring medicinal plants of India; journal of Ethano pharmacology, 114:103-113.

16. Guljar A, Singh MP, Singh A (2011) Wound healing potential of some medicinal plants, $136-145$.

17. Steenkamp V, Mathivha E, Gouws MC, Rensburg CEJ, (2004)Studies on antibacterial, antioxidant and fibroblast growth stimulation of wound healing remedies from South Afr. J. Ethnopharmacol, 95: 353-357.

18. Kanwar P, Sharma N, Rekha A (2006) Medicinal plants use in traditional health care system prevalent in Western Himalaya. India J. Tradit knowl, 5: 300-309.

19. Kumar B, Vinay KM, Govindarajan R, Pushpangadan P (2007) Ethano pharmacological approaches to wound healingexploring medicinal plants of India, J.Ethanopharmacol. 114:103-113.

20. Ayyanar M, Ignacimuthu S (2009) Herbal medicines for wound healing among tribal peoples in southern India : Ethnobotanical and Scientific evidences. International Journal of Applied Research in Natural Products 2: 29-42.

21. Muthu C, Ayyanar M, Raja N, Ignacimuthu S (2006) Medicinal plants used by traditional healers in Kancheepuram district of Tamil Nadu, India. Journal of Ethnobiology and Ethnomedicine 2: 43-53.

22. Ngari EW, Chiuri LW, Kariuki ST, Huckett S (2010) Ethnomedicine of Ogiek of River Njoro Watershed, Nakuru- Kenya. Ethnobot. Res. Appl, 8: 135-152.

23. Ratree M, Nathorn Chaiyakunapruk, Surachet Niruntraporn, Chuenjid Kongkaew (2007) The efficacy of Aloe vera used for burn wound healing:A systematic review, 33: 713-718.

24. Shila G, Natasa SB (2009) Wound healing properties of Carica papaya latex: In vivo evaluationin mice burn modelJournal of Ethnopharmacology 121: 338-341. 